\title{
THE HOMOTOPY SEQUENCE OF THE ALGEBRAIC FUNDAMENTAL GROUP
}

\author{
LEI ZHANG
}

\begin{abstract}
In this paper, we prove that the homotopy sequence of the algebraic fundamental group is exact if the base field is of characteristic 0. So in particular, the Künneth formula holds in characteristic 0 .
\end{abstract}

\section{INTRODUCTION}

Let $f: X \rightarrow S$ be a separable proper surjective morphism with geometrically connected fibres between locally noetherian connected schemes, $x \hookrightarrow X$ be a geometric point, $s=$ $f(x), X_{s}$ be the fibre at the geometric point $s$. Grothendieck proved in [SGA1, Exposé X, Corollaire 1.4] that there is a homotopy exact sequence for the étale fundamental group:

$$
\pi_{1}^{\text {ét }}\left(X_{s}, x\right) \rightarrow \pi_{1}^{\text {ét }}(X, x) \rightarrow \pi_{1}^{\text {ét }}(S, s) \rightarrow 1 .
$$

In particular, one can take $X, Y$ to be two locally noetherian connected $k$-schemes with $k=\bar{k}$ and assume that $Y$ is proper separable over $k$. If we take a $k$-point $z=(x, y)$ : $\operatorname{Spec}(k) \rightarrow X \times_{k} Y$, we can get a canonical homomorphism of group schemes

$$
\pi_{1}^{\text {ét }}\left(X \times_{k} Y, z\right) \rightarrow \pi_{1}^{\text {ét }}(X, x) \times \pi_{1}^{\text {ét }}(Y, y) .
$$

Applying the homotopy exact sequence we see that the canonical homomorphism is an isomorphism. This is called the Künneth formula for the étale fundamental group.

If $X$ is a smooth geometrically connected scheme over a field $k$ with a rational point $x \in X(k)$, we can consider the category of $O_{X}$-coherent $D_{X / k}$-modules $\operatorname{Mod}_{c}\left(D_{X / k}\right)$. Let $\omega_{x}$ be the functor $\operatorname{Mod}_{c}\left(D_{X / k}\right) \rightarrow \operatorname{Vec}_{k}$ sending any $O_{X}$-coherent $D_{X / k}$-module $M$ to $\left.M\right|_{x}$ (the restriction of $M$ at $x \in X)$. The category $\operatorname{Mod}_{c}\left(D_{X / k}\right)$ together with $\omega_{x}$ is a neutral Tannakian category, and its Tannakian group $\pi^{\text {alg }}(X, x)$ is defined to be the algebraic fundamental group of $(X, x) . \pi^{\text {alg }}(X, x)$ is functorial in $(X, x)$. So if we have a proper smooth map $f: X \rightarrow S$ between two smooth connected schemes over a field $k$ with geometrically connected fibres, if $x \in X(k)$ and $f(x)=s$, then we get a sequence of maps

$$
\pi^{\mathrm{alg}}\left(X_{s}, x\right) \rightarrow \pi^{\mathrm{alg}}(X, x) \rightarrow \pi^{\mathrm{alg}}(S, s) \rightarrow 1 .
$$

Theorem 1.1. Let $f: X \rightarrow S$ be a proper smooth morphism between two smooth connected schemes of finite type over a field $k$ of characteristic 0 . Suppose that all the geometric fibres

Date: April 15, 2012.

This work was supported by the Sonderforschungsbereich/Transregio 45 "Periods, moduli spaces and the arithmetic of algebraic varieties" of the DFG. 
of $f$ are connected. Let $x \in X(k), s \in S(k)$ and $f(x)=s$. Then the homotopy sequence (因) is exact.

In the proof, we first apply the exactness criterion 2.1 in the general Tannakian category theory to the neutral Tannakian category of $O_{X}$-coherent $D_{X / k}$-modules with a fibre functor induced by a "generic geometric point". We check that each condition of the criterion is satisfied in this situation, and deduce that the homotopy sequence is exact for schemes with a "generic geometric point". Then we use a little transcendental method to show that if the homotopy sequence is exact for schemes with a "generic geometric point" then it is exact for schemes with arbitrary $k$-rational base point. Since the category of $O_{X}$-coherent $D_{X / k}$-modules is the same as the category of $O_{X}$-coherent flat connections when the base field is of characteristic 0 , we are able to work with flat connections instead of the more complicated $D_{X / k}$-modules. For this reason the proof is hard to be generalized to positive characteristics. If $X$ is a scheme proper over $\mathbb{C}$, then the algebraic fundamental group is the algebraic completion of the topological fundamental group, so one might be tempted to use the homotopy exact sequence of the topological fundamental group to prove the theorem. However, it turns out that the algebraic completion functor does not behave well with respect to exactness, so we have to work with the category of flat connections instead. This makes our proof more algebraic.

A crucial part (§3.2) of the proof of this theorem is based on [E]. The main idea of that section is from the unpublished letter.

As a direct corollary of the homotopy exact sequence in characteristic 0 we have:

Corollary 1.2. Let $T$ and $S$ be smooth geometrically connected schemes over a field $k$ of characteristic 0. Assume that $T$ is proper over $k$. Then the canonical $k$-group scheme homomorphism

$$
\pi^{\mathrm{alg}}\left(T \times_{k} S,(t, s)\right) \rightarrow \pi^{\mathrm{alg}}(T, t) \times_{k} \pi^{\mathrm{alg}}(S, s)
$$

is an isomorphism. In other words, the Künneth formula holds in characteristic 0.

Acknowledgments: I would like to express my deepest gratitude to my advisor Hélène Esnault for proposing to me the questions and guiding me through this topic with patience, in fact a crucial part (§3.2) in the proof of the homotopy sequenece in characteristic 0 is from her idea. I thank my co-advisor Phùng Hô Hai for numerous helpful discussions.

\section{PRELiminaries}

2.1. The general criterion. In [EPS, Appendix Theorem A.1], Hélène Esnault, Phùng Hô Hai, Xiaotao Sun formulated a necessary and sufficient condition for the exactness of a sequence of Tannakian groups in terms of the corresponding tensor functors. Since the algebraic fundamental group is defined via Tannakian duality, to prove the exactness of the homotopy sequence (囵) we only need to check the condition for our specific category the category of $D$-modules. For the convenience of the reader we rewrite the condition in the following theorem. See [EPS, Appendix Theorem A.1] for details. 
Theorem 2.1. ([EPS, Appendix Theorem A.1]) Let $L \stackrel{q}{\rightarrow} G \stackrel{p}{\rightarrow} A$ be a sequence of homomorphisms of affine group schemes over a field $k$. It induces a sequence of functors:

$$
\operatorname{Rep}_{k}(A) \stackrel{p^{*}}{\rightarrow} \operatorname{Rep}_{k}(G) \stackrel{q^{*}}{\rightarrow} \operatorname{Rep}_{k}(L)
$$

where $\operatorname{Rep}_{k}(-)$ denotes the category of finite dimensional representations of - over $k$. Then we have

(1) The group homomorphism $p: G \rightarrow A$ is surjective (faithfully flat) if and only if $p^{*} \operatorname{Rep}_{k}(A)$ is a full subcategory of $\operatorname{Rep}_{k}(G)$ and closed under taking subquotients.

(2) The group homomorphism $q: L \rightarrow G$ is injective (a closed immersion) if and only if any object of $\operatorname{Rep}_{k}(L)$ is a subquotient of an object of the form $q^{*}(V)$ for some $V \in \operatorname{Rep}_{k}(G)$.

(3) Assume that $q$ is a closed immersion and that $p$ is faithfully flat. Then the sequence $L \stackrel{q}{\rightarrow} G \stackrel{p}{\rightarrow} A$ is exact if and only if the following conditions are fulfilled:

(a) For an object $V \in \operatorname{Rep}_{k}(G), q^{*} V \in \operatorname{Rep}_{k}(L)$ is trivial if and only if $V \cong p^{*} U$ for some $U \in \operatorname{Rep}_{k}(A)$

(b) Let $W_{0}$ be the maximal trivial subobject of $q^{*} V$ in $\operatorname{Rep}_{k}(L)$. Then there exists $V_{0} \subseteq V$ in $\operatorname{Rep}_{k}(G)$, such that $q^{*} V_{0} \cong W_{0}$.

(c) Any $W$ in $\operatorname{Rep}_{k}(L)$ is embeddable in $q^{*} V$ for some $V \in \operatorname{Rep}_{k}(G)$.

Remark 2.2. (I). The equivalence condition for surjectivity can also be written as:

Condition (1)'. $p: G \rightarrow A$ is surjective if and only if $p^{*}$ is fully faithful and $p^{*}\left(\operatorname{Rep}_{k}(A)\right)$ is stable under taking subobjects.

In fact, because $p^{*}$ is an exact functor, if $p^{*}\left(\operatorname{Rep}_{k}(A)\right)$ is stable under taking subobjects then it is stable under taking subquotients.

(II). In (3), if $q$ is not necessarily a closed immersion, then (c) should be replaced by:

Condition (c)'. For any $W^{\prime} \in \operatorname{Rep}_{k}(G)$ and any quotient $q^{*} W^{\prime} \rightarrow W \in \operatorname{Rep}_{k}(L)$, there exists $V \in \operatorname{Rep}_{k}(G)$ and an imbedding $W \hookrightarrow q^{*} V$.

In fact, we could decompose the homomorphism $q: L \rightarrow G$ into a composition $L \rightarrow$ $L^{\prime} \hookrightarrow G$. Let

$$
\operatorname{Rep}_{k}(G) \stackrel{a^{*}}{\rightarrow} \operatorname{Rep}_{k}\left(L^{\prime}\right) \stackrel{b^{*}}{\rightarrow} \operatorname{Rep}_{k}(L)
$$

be the corresponding tensor functors. By condition (1), (2) we know that $b^{*}$ is fully faithful and closed under taking subquotients and that any object in $\operatorname{Rep}_{k}\left(L^{\prime}\right)$ is a subquotient of $a^{*} W^{\prime}$ where $W^{\prime} \in \operatorname{Rep}_{k}(G)$. Since the exactness of $L \stackrel{q}{\rightarrow} G \stackrel{p}{\rightarrow} A \rightarrow 0$ is equivalent to the exactness of $0 \rightarrow L^{\prime} \stackrel{a}{\rightarrow} G \stackrel{p}{\rightarrow} A \rightarrow 0$, we could replace (c) by the condition that for any $W^{\prime} \in \operatorname{Rep}_{k}(G)$ and any subquotient $W$ of $a^{*} W^{\prime}$, there exists $V \in \operatorname{Rep}_{k}(G)$ and an imbedding $W \hookrightarrow a^{*} V$. Now one can check formally that this condition is the same as (c)'. 


\section{The homotopy EXACT SEquence in Characteristic 0}

In this section $k$ is always a field of characteristic 0 . In this case the category $\operatorname{Mod}_{c}\left(D_{X / k}\right)$ is the same as the category of vector bundles with flat connections, so in the following we will work purely in the category of vector bundles with flat connections and still use $\operatorname{Mod}_{c}\left(D_{X / k}\right)$ to denote this category.

\subsection{The conditions (a), (b) and the surjectivity.}

The Setup for $\S 3.1$. Let $f: X \rightarrow S$ be a smooth proper morphism with geometrically connected fibres between two smooth connected schemes of finite type over $k, s \in S(k)$ be a rational point, $X_{s}$ be the fibre, $x \in X(k)$ be a rational point lying above $s$, then by the functoriality of the algebraic fundamental group we get a sequence of affine group schemes

$$
\pi^{\mathrm{alg}}\left(X_{s}, x\right) \rightarrow \pi^{\mathrm{alg}}(X, x) \rightarrow \pi^{\mathrm{alg}}(S, s) \rightarrow 1,
$$

which is called the homotopy sequence. We will show that the sequence is exact by checking the conditions provided in 2.1.

Theorem 3.1. The homotopy sequence

$$
\pi^{\operatorname{alg}}\left(X_{s}, x\right) \rightarrow \pi^{\operatorname{alg}}(X, x) \rightarrow \pi^{\operatorname{alg}}(S, s) \rightarrow 1
$$

is a complex, and the arrow $\pi^{\mathrm{alg}}(X, x) \rightarrow \pi^{\mathrm{alg}}(S, s)$ is surjective.

Proof. Since $s \in S(k)$ is a rational point, the pull back of any object in $\operatorname{Mod}_{c}\left(D_{S / k}\right)$ is trivial in $\operatorname{Mod}_{c}\left(D_{X_{s} / k}\right)$, so the sequence is a complex. To show the surjectivity of right arrow, one has to show that the functor $f^{*}: \operatorname{Mod}_{c}\left(D_{S / k}\right) \rightarrow \operatorname{Mod}_{c}\left(D_{X / k}\right)$ is fully faithful and stable under taking subobject.

The fact that $f^{*}$ is fully faithful follows readily from the projection formula, so we only have to show that it is stable under taking subobject. Suppose that we have an object $\left(E, \nabla_{E}\right) \in \operatorname{Mod}_{c}\left(D_{S / k}\right)$, and a subobject $\left(F, \nabla_{F}\right) \hookrightarrow f^{*}\left(E, \nabla_{E}\right)$.

First of all, we claim that $f_{*} F$ is a locally free sheaf of rank equal to that of $F$, and the adjunction map $f^{*} f_{*} F \rightarrow F$ is an isomorphism. Moreover, the natural map $f_{*} F \rightarrow E$ imbeds $f_{*} F$ as a subbundle of $E$ (locally split). In fact, for any point $t \in S$, the restriction $\left.F\right|_{X_{t}}$ of $F$ to $X_{t}$ is a free $O_{X_{t}}$-module. This is because $\left.f^{*}\left(E, \nabla_{E}\right)\right|_{X_{t} / \kappa(t)}$ is a trivial object in $\operatorname{Mod}_{c}\left(D_{X_{t} / \kappa(t)}\right)$, and $\left.\left.\left(F, \nabla_{F}\right)\right|_{X_{t} / \kappa(t)} \subseteq f^{*}\left(E, \nabla_{E}\right)\right|_{X_{t} / \kappa(t)}$, so $\left.\left(F, \nabla_{F}\right)\right|_{X_{t} / \kappa(t)}$ has to be a trivial object too. This implies that $\left.F\right|_{X_{t}}$ is a free $O_{X_{t}}$-module. Thus, by [Mum, Page 48, Chapter 2, §5, Corollary 2], $f_{*} F$ satisfies base change in degree 0 at each point $t \in S$. Hence $f_{*} F$ is a vector bundle and its rank is equal to the rank of $F$. Since the maps $f^{*} f_{*} F \rightarrow F$ and $f_{*} F \rightarrow E$ are injective after restricting to each fibre $X_{t}$ with $t \in S$, it follows from the local criterion of flatness [Mats, Page 176, §22, Theorem 22.5] that they themselves are injective and their quotients are locally free (i.e. they are imbeddings of subbundles). Furthermore, since $f^{*} f_{*} F$ and $F$ have the same rank, the map $f^{*} f_{*} F \rightarrow F$ has to be an isomorphism. This finishes the proof of the claim.

Now from the connection $\nabla_{F}$, we get a map:

$$
f_{*} F \rightarrow f_{*}\left(F \otimes_{O_{X}} \Omega_{X / k}^{1}\right) \cong f_{*}\left(f^{*} f_{*} F \otimes_{O_{X}} \Omega_{X / k}^{1}\right) \cong f_{*} F \otimes_{O_{S}} f_{*} \Omega_{X / k}^{1} .
$$


Since $f: X \rightarrow S$ is smooth, the exact sequence

$$
0 \rightarrow f^{*} \Omega_{S / k}^{1} \rightarrow \Omega_{X / k}^{1} \rightarrow \Omega_{X / S}^{1} \rightarrow 0
$$

splits locally. Hence we have an induced injection

$$
f^{*}\left(E / f_{*} F\right) \otimes_{O_{X}} f^{*} \Omega_{S / k}^{1} \hookrightarrow f^{*}\left(E / f_{*} F\right) \otimes_{O_{X}} \Omega_{X / k}^{1}
$$

which is just

$$
E / f_{*} F \otimes_{O_{S}} \Omega_{S / k}^{1} \hookrightarrow E / f_{*} F \otimes_{O_{S}} f_{*} \Omega_{X / k}^{1}
$$

after applying $f_{*}$ and the projection formula. Now look at the following commutative diagram with exact rows:

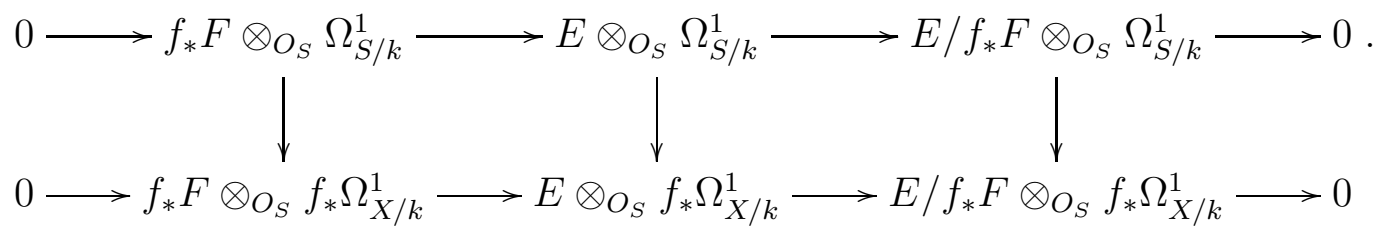

Since $f_{*} F$ maps to $f_{*} F \otimes_{O_{S}} f_{*} \Omega_{X / k}^{1}$, its image in $E / f_{*} F \otimes_{O_{S}} f_{*} \Omega_{X / k}^{1}$ is trivial. Because $E / f_{*} F \otimes_{O_{S}} \Omega_{S / k}^{1} \hookrightarrow E / f_{*} F \otimes_{O_{S}} f_{*} \Omega_{X / k}^{1}$ is injective, $f_{*} F \rightarrow E \otimes_{O_{S}} \Omega_{S / k}^{1}$ factors through $f_{*} F \otimes_{O_{S}} \Omega_{S / k}^{1}$. This proves that $f_{*} F \subseteq E$ is equipped with a flat connection $f_{*} \nabla_{F}$ which makes $\left(f_{*} F, f_{*} \nabla_{F}\right)$ a subobject of $\left(E, \nabla_{E}\right)$. Clearly $f^{*}\left(f_{*} F, f_{*} \nabla_{F}\right) \cong\left(F, \nabla_{F}\right)$ as subobjects of $f^{*}\left(E, \nabla_{E}\right)$. This finishes the proof.

Corollary 3.2. For any object $\left(E, \nabla_{E}\right) \in \operatorname{Mod}_{c}\left(D_{X / k}\right)$, the natural map

$$
\phi: f^{*} H_{D R}^{0}\left(X / S,\left(E, \nabla_{E}\right)\right)=f^{*} f_{*} E^{\nabla_{X / S}} \rightarrow E
$$

is horizontal (i.e. a morphism in $\operatorname{Mod}_{c}\left(D_{S / k}\right)$ ) with respect to the Gauss-Manin connection on the left. Moreover, $\phi$ is injective and imbeds $f^{*} H_{D R}^{0}\left(X / S,\left(E, \nabla_{E}\right)\right)$ as the "maximal pull back subobject" of $\left(E, \nabla_{E}\right)$ in the following sense:

If $\left(M, \nabla_{M}\right) \subseteq\left(E, \nabla_{E}\right) \in \operatorname{Mod}_{c}\left(D_{X / k}\right)$ such that $\left(M, \nabla_{M}\right)=f^{*}\left(N, \nabla_{N}\right)$ for some $\left(N, \nabla_{N}\right) \in \operatorname{Mod}_{c}\left(D_{S / k}\right)$, then the imbedding $\left(M, \nabla_{M}\right) \subseteq\left(E, \nabla_{E}\right)$ factors through $\phi$.

Proof. The fact that $\phi$ is horizontal is from the definition of the Gauss-Manin connection. To show that it is injective one considers the kernel $\left(K, \nabla_{K}\right)$ of the map. One has:

$$
0 \rightarrow\left(K, \nabla_{K}\right) \rightarrow f^{*} H_{D R}^{0}\left(X / S,\left(E, \nabla_{E}\right)\right) \stackrel{\phi}{\rightarrow}\left(E, \nabla_{E}\right)
$$

is exact. Since the functor $H_{D R}^{0}(X / S,-)$ is left exact and $H_{D R}^{0}(X / S, \phi)$ is an isomorphism, we have $H_{D R}^{0}\left(X / S,\left(K, \nabla_{K}\right)\right)=0$. But by the 3.1, one has an object $\left(K^{\prime}, \nabla_{K^{\prime}}\right) \in$ $\operatorname{Mod}_{c}\left(D_{S / k}\right)$ such that $f^{*}\left(K^{\prime}, \nabla_{K^{\prime}}\right)=\left(K, \nabla_{K}\right)$. Thus as sheaves on $S$, one has $0=$ $H_{D R}^{0}\left(X / S,\left(K, \nabla_{K}\right)\right)=H_{D R}^{0}\left(X / S, f^{*}\left(K^{\prime}, \nabla_{K^{\prime}}\right)\right) \cong K^{\prime}$. This shows that $K=0$. Thus $\phi$ is injective.

Suppose $\left(M, \nabla_{M}\right) \subseteq\left(E, \nabla_{E}\right) \in \operatorname{Mod}_{c}\left(D_{X / k}\right)$ such that $\left(M, \nabla_{M}\right)=f^{*}\left(N, \nabla_{N}\right)$ for some $\left(N, \nabla_{N}\right) \in \operatorname{Mod}_{c}\left(D_{S / k}\right)$, then $N \cong f_{*} M^{\nabla_{X / S}} \hookrightarrow f_{*} E^{\nabla_{X / S}}=H_{D R}^{0}\left(X / S,\left(E, \nabla_{E}\right)\right)$. This shows that $M \hookrightarrow E$ factors through $\phi$. 
Theorem 3.3. For any $\left(E, \nabla_{E}\right) \in \operatorname{Mod}_{c}\left(D_{X / k}\right)$ the restriction of the subobject

$$
\left(F, \nabla_{F}\right):=f^{*} H_{D R}^{0}\left(X / S,\left(E, \nabla_{E}\right)\right) \hookrightarrow\left(E, \nabla_{E}\right)
$$

to $X_{s}$ gives the maximal trivial subobject of $\left.\left(E, \nabla_{E}\right)\right|_{X_{s} / k}$. So in particular, the condition (a) and (b) of 2.1 (3) are satisfied.

Proof. Since the maximal trivial subobject of $\left.\left(E, \nabla_{E}\right)\right|_{X_{s} / k}$ is precisely

$$
\left.f^{*} H_{D R}^{0}\left(X_{s} / k,\left.\left(E, \nabla_{E}\right)\right|_{X_{s} / k}\right) \hookrightarrow\left(E, \nabla_{E}\right)\right|_{X_{s} / k},
$$

the theorem follows from the base change theorem for the Gauss-Manin connection (See, for example, [Katz, Section 8]).

3.2. The condition (c) for a generic geometric point. Now we come to check the condition (c) in the general criterion. Since we are not going to show the injectivity of the very left arrow, the condition (c) in our situation reads (see 2.2):

For any $\left(E, \nabla_{E}\right) \in \operatorname{Mod}_{c}\left(D_{X / k}\right)$ and any quotient

$$
\left.\left(E, \nabla_{E}\right)\right|_{X_{s} / k} \rightarrow\left(F^{\prime}, \nabla_{F^{\prime}}\right) \in \operatorname{Mod}_{c}\left(D_{X_{s} / k}\right),
$$

there exists $\left(F, \nabla_{F}\right) \in \operatorname{Mod}_{c}\left(D_{X / k}\right)$ and an imbedding

$$
\left.\left(F^{\prime}, \nabla_{F^{\prime}}\right) \hookrightarrow\left(F, \nabla_{F}\right)\right|_{X_{s} / k} \in \operatorname{Mod}_{c}\left(D_{X_{s} / k}\right) .
$$

Or equivalently, by taking dual, one can say that for any $\left(E, \nabla_{E}\right) \in \operatorname{Mod}_{c}\left(D_{X / k}\right)$ and any subobject $\left.\left(F^{\prime}, \nabla_{F^{\prime}}\right) \hookrightarrow\left(E, \nabla_{E}\right)\right|_{X_{s} / k} \in \operatorname{Mod}_{c}\left(D_{X_{s} / k}\right)$, there exists $\left(F, \nabla_{F}\right) \in \operatorname{Mod}_{c}\left(D_{X / k}\right)$ and a surjection $\left.\left(F, \nabla_{F}\right)\right|_{X_{s} / k} \rightarrow\left(F^{\prime}, \nabla_{F^{\prime}}\right) \in \operatorname{Mod}_{c}\left(D_{X_{s} / k}\right)$.

This condition here is quite difficult to check, but since (a) and (b) are satisfied, (c) is now equivalent to the exactness of the homotopy sequence. We will first prove this condition in a special case (for a "generic geometric point") then we will show that if in this special case the condition holds then it holds in general. Next we will place the settings for the case of a "generic geometric point".

The Setup for $\S 3.2$. Let $f_{0}: X_{0} \rightarrow S_{0}$ be a smooth morphism between smooth geometrically connected schemes of finite type over a field $k_{0}$ of characteristic 0 . Let $k$ be an algebraic extension of the function field $\kappa\left(S_{0}\right)$ of $S_{0}, S:=S_{0} \times_{k_{0}} k, X:=X_{0} \times_{k_{0}} k$, $f:=f_{0} \times_{k_{0}} k$. Then we get a $k$-rational point $s \in S$ which corresponds to the generic point of $S_{0}$. This point is called the generic geometric point of $S_{0}$. Let $X_{s}$ be the fibre of $f$ at $s \in S(k)$. Assume there is $x \in X(k)$ such that $f(x)=s$.
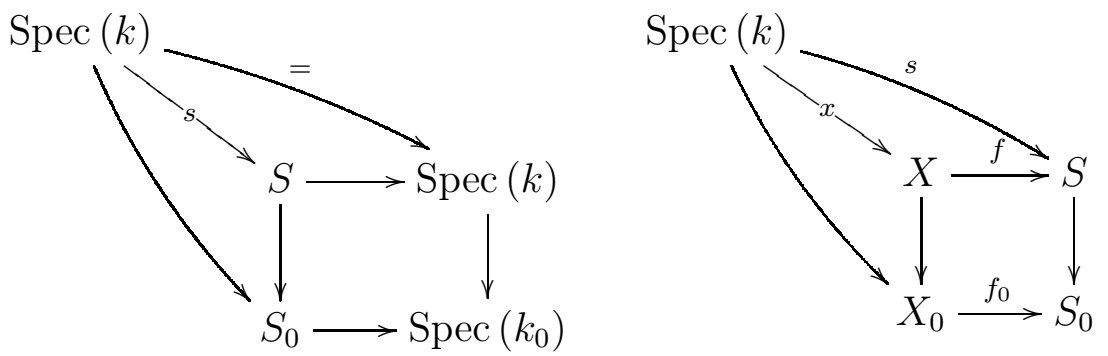
Proposition 3.4. If $\left(E, \nabla_{E}\right) \in \operatorname{Mod}_{c}\left(D_{X / k}\right),\left.\left(F^{\prime}, \nabla_{F^{\prime}}\right) \subseteq\left(E, \nabla_{E}\right)\right|_{X_{s} / k} \in \operatorname{Mod}_{c}\left(D_{X_{s} / k}\right)$, then $\exists$ a non-trivial Zariski open $U_{0} \subseteq S_{0}$ and an object $\left(F_{0}, \nabla_{F_{0}}\right) \in \operatorname{Mod}_{c}\left(D_{f_{0}^{-1}\left(U_{0}\right) / k_{0}}\right)$ with a surjection $\left.\left(F_{0}, \nabla_{F_{0}}\right)\right|_{X_{s} / k} \rightarrow\left(F^{\prime}, \nabla_{F^{\prime}}\right)$.

Proof. According to 3.5 below, we have a non-trivial Zariski open $U_{0} \subseteq S_{0}$ and a finite étale covering $T_{0} \rightarrow U_{0}$ with $\kappa\left(T_{0}\right) \subseteq k$ such that $\left(E, \nabla_{E}\right) \in \operatorname{Mod}_{c}\left(D_{X / k}\right)$ is defined over $\operatorname{Mod}_{c}\left(D_{X_{0} \times_{k_{0}} T_{0} / T_{0}}\right)$. We may assume $U_{0}=S_{0}$ and let $\left(E_{0}, \nabla_{E_{0}}\right) \in \operatorname{Mod}_{c}\left(D_{X_{0} \times k_{0} T_{0} / T_{0}}\right)$ be the object such that $\rho^{*}\left(E_{0}, \nabla_{E_{0}}\right) \cong\left(E, \nabla_{E}\right)$ where $\rho: X=X_{0} \times_{k_{0}} k \rightarrow X_{0} \times_{k_{0}} T_{0}$. Let $\alpha: T_{0} \hookrightarrow S_{0} \times_{k_{0}} T_{0}$ be the graph of $T_{0} \rightarrow S_{0}$ and $\beta: Z_{T_{0}} \hookrightarrow X_{0} \times_{k_{0}} T_{0}$ be the pull back of the graph:

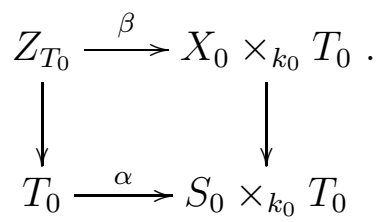

Then the pull back $\beta^{*}\left(E_{0}, \nabla_{E_{0}}\right) \in \operatorname{Mod}_{c}\left(D_{Z_{T_{0}} / T_{0}}\right)$ is actually defined over $\operatorname{Mod}_{c}\left(D_{Z_{T_{0}} / k_{0}}\right)$. In fact, we have the following commutative diagram:

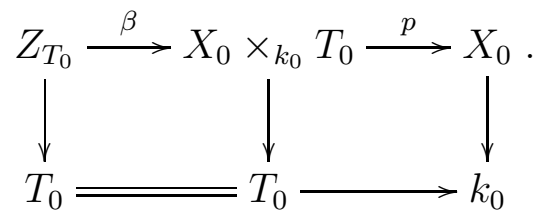

Thus we have maps

$$
\beta^{*} \Omega_{X_{0} \times k_{0} T_{0} / T_{0}}^{1} \cong \beta^{*} p^{*} \Omega_{X_{0} / k_{0}}^{1} \rightarrow \Omega_{Z_{T_{0}} / k_{0}}^{1} .
$$

Note that the last arrow in the above sequence is actually coming from the following commutative diagramme:

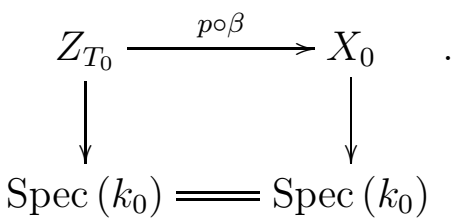

This indeed extends our connection

$$
\nabla_{E_{0}}: E_{0} \rightarrow E_{0} \otimes_{O_{X_{0} \times S_{0} T_{0}}} \Omega_{X_{0} \times_{k_{0}} T_{0} / T_{0}}^{1} \cong E_{0} \otimes_{O_{X_{0} \times S_{0} T_{0}}} p^{*} \Omega_{X_{0} / k_{0}}^{1}
$$

to the connection

$$
\beta^{*} \nabla_{E_{0}}: \beta^{*} E_{0} \rightarrow \beta^{*} E_{0} \otimes_{O_{Z_{T_{0}}}} \Omega_{Z_{T_{0}} / k_{0}}^{1} .
$$

Let $\lambda: Z_{T_{0}} \rightarrow Z_{S_{0}} \cong X_{0}$. Since $T_{0} \rightarrow S_{0}$ is finite étale, we have $\lambda_{*} \beta^{*}\left(E_{0}, \nabla_{E_{0}}\right) \in$ $\operatorname{Mod}_{c}\left(D_{X_{0} / k_{0}}\right)$, and there is a surjection

$$
\lambda^{*} \lambda_{*} \beta^{*}\left(E_{0}, \nabla_{E_{0}}\right) \rightarrow \beta^{*}\left(E_{0}, \nabla_{E_{0}}\right) .
$$


From the Cartesian diagrams

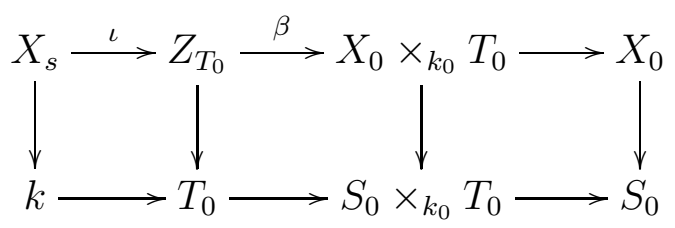

we know that if we pull back $\beta^{*}\left(E_{0}, \nabla_{E_{0}}\right)$ along $\iota$ then we get $\left.\left(E, \nabla_{E}\right)\right|_{X_{s} / k}$. Now let $\left(F^{\prime \prime}, \nabla_{F^{\prime \prime}}\right)$ be the inverse image of $\left(F^{\prime}, \nabla_{F^{\prime}}\right)$ under the map

$$
\left.\left.\lambda^{*} \lambda_{*} \beta^{*}\left(E_{0}, \nabla_{E_{0}}\right)\right|_{X_{s} / k} \rightarrow \beta^{*}\left(E_{0}, \nabla_{E_{0}}\right)\right|_{X_{s} / k}=\left.\left(E, \nabla_{E}\right)\right|_{X_{s} / k} .
$$

According to 3.6 below, there exists a non-trivial Zariski open $U_{0} \subseteq S_{0}$ and $\left(F_{0}, \nabla_{F_{0}}\right) \in$ $\operatorname{Mod}_{c}\left(D_{f_{0}^{-1}\left(U_{0}\right) / k_{0}}\right)$ with a surjection $\left.\left(F_{0}, \nabla_{F_{0}}\right)\right|_{X_{s} / k} \rightarrow\left(F^{\prime \prime}, \nabla_{F^{\prime \prime}}\right) \rightarrow\left(F^{\prime}, \nabla_{F^{\prime}}\right) \in \operatorname{Mod}_{c}\left(D_{X_{s} / k}\right)$. This completes the proof.

Lemma 3.5. (The notations and conventions in this lemma are independent) Let $f$ : $X \rightarrow S$ be a smooth morphism between two integral noetherian schemes. Let $s \in S$ be the generic point, $\kappa(s) \subseteq k$ be a separable algebraic extension of fields, $X_{k}$ be the generic fibre (corresponding to $\operatorname{Spec}(k) \hookrightarrow S$ ). Then for any object $\left(F, \nabla_{F}\right) \in \operatorname{Mod}_{c}\left(D_{X_{k} / k}\right)$ with $F$ a vector bundle, there exists a non-empty open subset $U \subseteq S$, an integral finite étale covering $T \rightarrow U$ and an object $\left(E, \nabla_{E}\right) \in \operatorname{Mod}_{c}\left(D_{X \times{ }_{S} T / T}\right)$ which satisfy (1) the function field of $T$ is contained in $k$; (2) $\left(F, \nabla_{F}\right) \cong\left(E, \nabla_{E}\right)_{X_{k} / k}$.

Proof. Let $\phi: X_{k} \rightarrow X$ be the canonical imbedding of the generic fibre and assume $S=\operatorname{Spec}(R)$. Then we get a surjection $\phi^{*} \phi_{*} F \rightarrow F$. Since $\phi_{*} F$ is the union of its coherent subsheaves, we find a coherent subsheaf $M$ of $\phi_{*} F$ with a surjection $\phi^{*} M \rightarrow F$. Suppose $N \subseteq \phi^{*} M$ is the kernel of $\phi^{*} M \rightarrow F$. It is coherent because $X$ is noetherian. Then we can collect finitely many elements $\left\{x_{0}, \cdots, x_{n}\right\}$ in $k$ which are integral over $R$ and a non-zero element $f \in R$ such that $N$ is defined over $R_{1}:=R_{f}\left[x_{0}, \cdots, x_{n}\right]$. Thus $F$ is defined over $R_{1}$. Suppose that $E_{1}$ is a coherent sheaf on $X \times_{R} R_{1}$ such that $\rho_{1}^{*} E_{1} \cong F$, where $\rho_{1}: X_{k}=X \times_{R} k \rightarrow X \times_{R} R_{1}$. Since the problem is local for $S$, and $F$ is locally free, we may assume $E_{1}$ is locally free too. Then the map

$$
E_{1} \otimes_{O_{X \times R_{R}}} \Omega_{X \times_{R} R_{1} / R_{1}}^{1} \rightarrow \rho_{1 *} \rho_{1}^{*}\left(E_{1} \otimes_{O_{X \times{ }_{R} R_{1}}} \Omega_{X \times_{R} R_{1} / R_{1}}^{1}\right)
$$

is injective. Since the $k$-linear map

$$
\nabla_{F}: F \rightarrow F \otimes_{O_{X_{k}}} \Omega_{X_{k} / k}^{1}
$$

can be seen as a map

$$
\rho_{1}^{*} E_{1} \rightarrow \rho_{1}^{*}\left(E_{1} \otimes_{O_{X \times R_{R} R_{1}}} \Omega_{X \times_{R} R_{1} / R_{1}}^{1}\right),
$$

we can collect finite many elements $\left\{y_{0}, \cdots, y_{n}\right\}$ in $k$ which are integral over $R$ and a non-zero element $g \in R$ such that $\nabla_{F}$ is defined over $R_{2}=\left(R_{1}\right)_{g}\left[y_{0}, \cdots, y_{n}\right]$ as a flat connection. Thus we have found $T_{2}:=\operatorname{Spec} R_{2}$ and $\left(E_{2}, \nabla_{E_{2}}\right) \in \operatorname{Mod}_{c}\left(D_{X \times{ }_{S} T_{2} / T_{2}}\right)$ such that $\rho_{2}^{*}\left(E_{2}, \nabla_{E_{2}}\right) \cong\left(F, \nabla_{F}\right)$ (where $\left.\rho_{2}: X \times_{S} k \rightarrow X \times_{S} T_{2}\right)$ and the generic point of $T_{2}$ is a finite field extension of $\kappa(s)$. Now the map $T_{2} \rightarrow S$ which is finite onto its image is 
étale at the generic point of $T_{2}$, thus we get a non-empty open subset $T$ of $T_{2}$ such that $T$ is finite étale over some non-empty open $U$ of $S$. This is precisely what we want.

Lemma 3.6. For any object $\left(E, \nabla_{E}\right) \in \operatorname{Mod}_{c}\left(D_{X_{0} / k_{0}}\right)$ and any imbedding $\left(F^{\prime}, \nabla_{F^{\prime}}\right) \hookrightarrow$ $\left.\left(E, \nabla_{E}\right)\right|_{X_{s} / k} \in \operatorname{Mod}_{c}\left(D_{X_{s} / k}\right)$ there is a non-empty open $U_{0} \subseteq S_{0}$ and an object $\left(F, \nabla_{F}\right) \in$ $\operatorname{Mod}_{c}\left(D_{f^{-1}\left(U_{0}\right) / k_{0}}\right)$ which admits a surjection

$$
\left.\left.\left(F, \nabla_{F}\right)\right|_{X_{s} / k} \rightarrow\left(F^{\prime}, \nabla_{F^{\prime}}\right) \hookrightarrow\left(E, \nabla_{E}\right)\right|_{X_{s} / k} \in \operatorname{Mod}_{c}\left(D_{X_{s} / k}\right) .
$$

Proof. First suppose $\kappa\left(S_{0}\right) \subseteq k$ is a trivial extension. Let $r:=\operatorname{dim}_{O_{X_{s}}}\left(F^{\prime}\right)$. According to $\left[\mathrm{EP}\right.$, Theorem 5.10] we have a subobject $\left.\left(M, \nabla_{M}\right) \subseteq\left(E, \nabla_{E}\right)\right|_{X_{s} / k_{0}} \in \operatorname{Mod}_{c}\left(D_{X_{s} / k_{0}}\right)$ with a surjection $\left.\left(M, \nabla_{M}\right)\right|_{X_{s} / k} \rightarrow \operatorname{det}\left(F^{\prime}, \nabla_{F^{\prime}}\right)$. If we set $\left(F_{1}, \nabla_{F_{1}}\right):=\left(M, \nabla_{M}\right) \otimes_{O_{X_{s}}}$ $\left(\left.\wedge^{r-1}\left(E, \nabla_{E}\right)\right|_{X_{s} / k_{0}}\right)^{\vee}$, then it is a subobject

$$
\left.\left(F_{1}, \nabla_{F_{1}}\right) \subseteq\left(E, \nabla_{E}\right)\right|_{X_{s} / k_{0}} \otimes_{O_{X_{s}}}\left(\left.\wedge^{r-1}\left(E, \nabla_{E}\right)\right|_{X_{s} / k_{0}}\right)^{\vee} \in \operatorname{Mod}_{c}\left(D_{X_{s} / k_{0}}\right)
$$

with a surjection

$$
\left.\left(F_{1}, \nabla_{F_{1}}\right)\right|_{X_{s} / k} \rightarrow\left(F^{\prime}, \nabla_{F^{\prime}}\right) \cong \operatorname{det}\left(F^{\prime}, \nabla_{F^{\prime}}\right) \otimes_{O_{X_{s}}}\left(\wedge^{r-1}\left(F^{\prime}, \nabla_{F^{\prime}}\right)\right)^{\vee} .
$$

Let $u: X_{s} \rightarrow X_{0}$ be the canonical imbedding, then we take the inverse image of $u_{*} F_{1}$ under the canonical map

$$
E \otimes_{O_{X}}\left(\wedge^{r-1} E\right)^{\vee} \rightarrow u_{*} u^{*}\left(E \otimes_{O_{X}}\left(\wedge^{r-1} E\right)^{\vee}\right)
$$

and denote it by $F_{2}$. One can check there is a non-empty open subscheme $U_{0} \subseteq S_{0}$ so that $F_{2}$ is equipped with a flat connection on $f^{-1}\left(U_{0}\right) / k_{0}$ and becomes a subobject

$$
\left.\left(F_{2}, \nabla_{F_{2}}\right) \subseteq\left(\left(E, \nabla_{E}\right) \otimes_{O_{X_{0}}}\left(\wedge^{r-1}\left(E, \nabla_{E}\right)\right)^{\vee}\right)\right|_{f^{-1}\left(U_{0}\right) / k_{0}} \in \operatorname{Mod}_{c}\left(D_{f^{-1}\left(U_{0}\right) / k_{0}}\right)
$$

which satisfies $\left.\left(F_{2}, \nabla_{F_{2}}\right)\right|_{X_{s} / k_{0}} \cong\left(F_{1}, \nabla_{F_{1}}\right)$. This finishes the special case.

Now suppose $\kappa\left(S_{0}\right) \subseteq k$ is a non-trivial extension. It is clear that the map $\left(F^{\prime}, \nabla_{F^{\prime}}\right) \hookrightarrow$ $\left.\left(E, \nabla_{E}\right)\right|_{X_{s} / k}$ is defined over $\operatorname{Mod}_{c}\left(D_{X_{k^{\prime}} / k^{\prime}}\right)$ where $k^{\prime}$ is a finite extension of $\kappa\left(S_{0}\right)$ and $X_{k^{\prime}}:=$ $X_{0} \times_{S_{0}} k^{\prime}$. Thus we may assume $k / \kappa\left(S_{0}\right)$ is finite. Then the map $\alpha: X_{s} \rightarrow X_{0} \times_{S_{0}} \kappa\left(S_{0}\right)$ is finite étale. So we get a surjection

$$
\alpha^{*} \alpha_{*}\left(F^{\prime}, \nabla_{F^{\prime}}\right) \rightarrow\left(F^{\prime}, \nabla_{F^{\prime}}\right) \in \operatorname{Mod}_{c}\left(D_{X_{s} / k}\right)
$$

and an imbedding

$$
\alpha_{*}\left(F^{\prime}, \nabla_{F^{\prime}}\right) \hookrightarrow \alpha_{*}\left(\left.\left(E, \nabla_{E}\right)\right|_{X_{s} / k}\right) \in \operatorname{Mod}_{c}\left(D_{X_{0} \times_{S_{0}} \kappa\left(S_{0}\right) / \kappa\left(S_{0}\right)}\right) .
$$

Thus it is enough to show that $\alpha_{*}\left(\left.\left(E, \nabla_{E}\right)\right|_{X_{s} / k}\right)$ is defined in $\operatorname{Mod}_{c}\left(D_{f^{-1}\left(U_{0}\right) / k_{0}}\right)$ with $U_{0} \subseteq$ $S_{0}$ non-trivial Zariski open, since then we can apply the special case we discussed above to get a surjection on $\alpha_{*}\left(F^{\prime}, \nabla_{F^{\prime}}\right)$ from some object in $\operatorname{Mod}_{c}\left(D_{f^{-1}\left(U_{0}\right) / k_{0}}\right)$. Since the problem is local on $S_{0}$ we may assume $S_{0}=\operatorname{Spec}(R)$. Then one can find a finite ring extension $R \subseteq R^{\prime} \subseteq k$ such that $R^{\prime}$ has quotient field $k$ (e.g., the integral closure of $R$ in $k$ ). Again because our problem is local on $S_{0}$, one may assume $R^{\prime} / R$ is finite étale. Let $\beta: X_{0}^{\prime}:=X_{0} \times_{\operatorname{Spec}(R)} \operatorname{Spec}\left(R^{\prime}\right) \rightarrow X_{0}, u: X_{0} \times_{S_{0}} \kappa\left(S_{0}\right) \rightarrow X_{0}$. Then $u^{*} \beta_{*} \beta^{*}\left(E, \nabla_{E}\right) \cong$ $\alpha_{*}\left(\left.\left(E, \nabla_{E}\right)\right|_{X_{s} / k}\right)$, but $\beta_{*} \beta^{*}\left(E, \nabla_{E}\right) \in \operatorname{Mod}_{c}\left(D_{X_{0} / k_{0}}\right)$. This completes the proof. 
Definition 3.7. Let $\operatorname{Mod}_{c}\left(D_{S / k}, s\right)$ be the category whose objects are of the form $(U, M)$, where $U$ is an open subset of $S$ containing $s$ and $M$ is a coherent sheaf on $U$ with a flat connection $\nabla_{M}$ on $U / k$, whose morphisms between two objects $(U, M)$ and $\left(U^{\prime}, M^{\prime}\right)$ are defined by

$$
\operatorname{Mor}\left((U, M),\left(U^{\prime}, M^{\prime}\right)\right):=\operatorname{Hom}_{U \cap U^{\prime}}\left(\left.(U, M)\right|_{U \cap U^{\prime}},\left.\left(U^{\prime}, M^{\prime}\right)\right|_{U \cap U^{\prime}}\right) .
$$

Let $\operatorname{Mod}_{c}\left(D_{X / S / k}, f, s\right)$ be the category whose objects are of the form $(U, M)$, where $U$ is an open subset of $S$ containing $s$ and $M$ is a coherent sheaf on $f^{-1}(U)$ with a flat connection $\nabla_{M}$ on $f^{-1}(U) / k$, whose morphisms between two objects $(U, M)$ and $\left(U^{\prime}, M^{\prime}\right)$ are defined by

$$
\operatorname{Mor}\left((U, M),\left(U^{\prime}, M^{\prime}\right)\right):=\operatorname{Hom}_{f^{-1}\left(U \cap U^{\prime}\right)}\left(\left.(U, M)\right|_{f^{-1}\left(U \cap U^{\prime}\right)},\left.\left(U^{\prime}, M^{\prime}\right)\right|_{f^{-1}\left(U \cap U^{\prime}\right)}\right) .
$$

Proposition 3.8. Let $X$ be a smooth geometrically connected scheme of finite type over a field $k$ of characteristic $0, U \subseteq X$ be a dense open subscheme, then for any two objects $\left(E, \nabla_{E}\right),\left(F, \nabla_{F}\right) \in \operatorname{Mod}_{c}\left(D_{X / k}\right)$ and any morphism $f_{U}:\left.\left.\left(E, \nabla_{E}\right)\right|_{U / S} \rightarrow\left(F, \nabla_{F}\right)\right|_{U / k} \in$ $\operatorname{Mod}_{c}\left(D_{U / k}\right)$, we can uniquely extend $f_{U}$ to a morphism

$$
f:\left(E, \nabla_{E}\right) \rightarrow\left(F, \nabla_{F}\right) \in \operatorname{Mod}_{c}\left(D_{X / k}\right) .
$$

Proof. The uniqueness is clear: if we have two extensions $f$ and $f^{\prime}$ then the set of points on which $f=f^{\prime}$ is closed in $X$, but it already contains an open dense subset, so it has to be the whole of $X$. Thus by 3.9 we may assume $f_{U}$ is an isomorphism.

Now suppose for any point $x \in X \backslash U$ we can extend $f_{U}$ to a neighborhood of $x$, then using Zorn's lemma we can extend $f_{U}$ to a map on $X$. Hence the problem is local. We may assume $X=\operatorname{Spec}(A)$ to be a smooth integral $k$-algebra with an étale coordinate $X \rightarrow \mathbb{A}_{k}^{r}$ $(r=\operatorname{dim} X)$, and $E=F=A^{\oplus n}$. Let $K$ be the fraction field of $A$. The local map $f_{U}$ provides us with a commutative diagram:

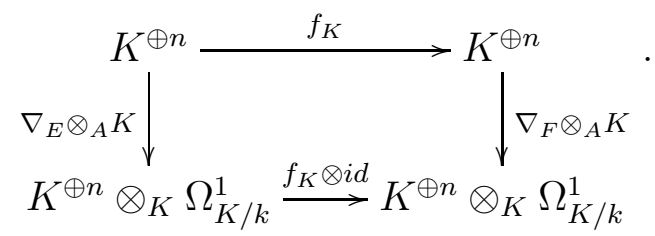

We need to show that $f_{K}$ takes $A^{\oplus n} \subseteq K^{\oplus n}$ to $A^{\oplus n} \subseteq K^{\oplus n}$. Or, equivalently, $f_{K}$ takes any vector $e_{i} \in K^{\oplus n}$ whose $i$-th entry is 1 and other entries are 0 to a vector whose entries are regular for any codimension 1 point $p \in \operatorname{Spec}(A)$.

For this purpose, we fix a codimension 1 point $p \in \operatorname{Spec}(A), \pi$ a uniformizer in $A_{p}$. Let $\hat{A}_{p}$ be the completion of $A_{p}, \hat{K}$ be the fraction field of $\hat{A}_{p}$. It suffices to check that the base change map $f_{\hat{K}}$ of $f_{K}$ sends $\hat{A}_{p}^{\oplus n}$ to $\hat{A}_{p}^{\oplus n}$. By [Katz, Proposition 8.9] we know that the connections $\hat{\nabla}_{E}$ and $\hat{\nabla}_{F}$ induced from $\nabla_{E}$ and $\nabla_{F}$ via base change $A \subseteq \hat{A}_{p}$ are trivial connections. This implies $f_{\hat{K}}\left(e_{i}\right)$ is a horizontal section for the connection $\hat{\nabla}_{F} \otimes_{\hat{A}_{p}} \hat{K}=d_{\hat{K}}^{\oplus n}$. Suppose there is an entry $w_{j}$ of $f_{\hat{K}}\left(e_{i}\right)$ which has a pole, i.e. $w_{j}=a \pi^{-k}$ where $a \in \hat{A}_{p}$ is invertible and $k$ a positive integer. Then we have

$$
d_{\hat{K}}\left(w_{j}\right)=\pi^{-k} d_{\hat{K}}(a)-k a \pi^{-k-1} d_{\hat{K}}(\pi) .
$$


Since $d_{\hat{K}}(a)$ is a regular 1 -form and $k$ is of characteristic $0, d_{\hat{K}}\left(w_{j}\right)=0$ would imply $\pi^{-1} d(\pi)$ is a regular 1 -form. This is absurd.

Lemma 3.9. Let $X$ be a smooth $k$-scheme, $U \subseteq X$ be an open subset, $\left(E, \nabla_{E}\right) \in$ $\operatorname{Mod}_{c}\left(D_{X / k}\right)$ and suppose that there is an injection

$$
\left.\left(F^{\prime}, \nabla_{F}^{\prime}\right) \hookrightarrow\left(E, \nabla_{E}\right)\right|_{U / k} \in \operatorname{Mod}_{c}\left(D_{U / k}\right)
$$

Then there exists a subobject $\left(F, \nabla_{F}\right) \subseteq\left(E, \nabla_{E}\right) \in \operatorname{Mod}_{c}\left(D_{X / k}\right)$ such that $\left.\left(F, \nabla_{F}\right)\right|_{U / k}=$ $\left(F^{\prime}, \nabla_{F}^{\prime}\right)$ as subojects of $\left.\left(E, \nabla_{E}\right)\right|_{U / k}$

Proof. Let $j: U \subseteq X$ be the inclusion. We take $F$ to be the inverse image of $j_{*} F^{\prime}$ under the adjunction map $E \rightarrow j_{*} j^{*} E$. Then $F$ is a coherent sheaf, and one checks easily that $F \rightarrow E \stackrel{\nabla_{E}}{\longrightarrow} E \times_{O_{X}} \Omega_{X / k}^{1}$ factors through $F \times_{O_{X}} \Omega_{X / k}^{1} \rightarrow E \times_{O_{X}} \Omega_{X / k}^{1}$. Hence $F$ is equipped with a connection $\nabla_{F}$ and becomes a subobject of $\left(E, \nabla_{E}\right)$. Clearly $\left.\left(F, \nabla_{F}\right)\right|_{U / k}$ is equal to $\left(F^{\prime}, \nabla_{F^{\prime}}\right)$ as subojects (since $\left.F\right|_{U}$ is equal to $F^{\prime}$ ).

3.8 and 3.9 imply immediately the following:

Lemma 3.10. The category $\operatorname{Mod}_{c}\left(D_{S / k}, S\right)\left(\operatorname{resp.} \operatorname{Mod}_{c}\left(D_{X / S / k}, f, s\right)\right)$ is an abelian $k$ linear rigid tensor category equipped with an exact faithful $k$-linear tensor functor $(M, U) \mapsto$ $\left.M\right|_{s}\left(\right.$ resp. $\left.\left.(M, U) \mapsto M\right|_{x}\right)$, i.e. a neutral Tannakian category. So we have a Tannakian group $\hat{\pi}^{\text {alg }}(S, s)\left(\right.$ resp. $\left.\hat{\pi}^{\text {alg }}(X, x)\right)$ associated to $\operatorname{Mod}_{c}\left(D_{S / k}, s\right)\left(\operatorname{resp} . \operatorname{Mod}_{c}\left(D_{X / S / k}, f, s\right)\right)$. Furthermore, the canonical functor $\operatorname{Mod}_{c}\left(D_{S / k}\right) \rightarrow \operatorname{Mod}_{c}\left(D_{S / k}, s\right)\left(\right.$ resp. $\operatorname{Mod}_{c}\left(D_{X / k}\right) \rightarrow$ $\left.\operatorname{Mod}_{c}\left(D_{X / S / k}, f, s\right)\right)$ is fully faithful and stable under taking subquotients. Thus we get a canonical surjection $\hat{\pi}^{\text {alg }}(S, s) \rightarrow \pi^{\text {alg }}(S, s)\left(\right.$ resp. $\left.\hat{\pi}^{\text {alg }}(X, x) \rightarrow \pi^{\text {alg }}(X, x)\right)$.

Using results in $§ 3.1$ and applying 3.4 to $\operatorname{Mod}_{c}\left(D_{X / S / k}, f, s\right)$ and $\operatorname{Mod}_{c}\left(D_{S / k}, s\right)$ we get:

Theorem 3.11. The homotopy sequence

$$
\pi^{a l g}\left(X_{s}, x\right) \rightarrow \hat{\pi}^{\text {alg }}(X, x) \rightarrow \hat{\pi}^{\text {alg }}(S, s) \rightarrow 1
$$

is exact. Moreover, there is a commutative diagram

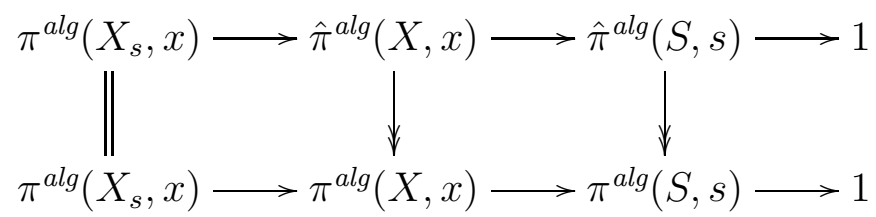

where all the vertical arrows are surjective.

Theorem 3.12. The homotopy sequence

$$
\pi^{a l g}\left(X_{s}, x\right) \rightarrow \pi^{a l g}(X, x) \rightarrow \pi^{a l g}(S, s) \rightarrow 1
$$

is exact. 
Proof. From the surjectivity of $\hat{\pi}^{\text {alg }}(X, x) \rightarrow \pi^{\text {alg }}(X, x)$ we know that the image of $\pi^{\text {alg }}\left(X_{s}, x\right)$ is a normal subgroup of $\pi^{\mathrm{alg}}(X, x)$. Taking $G:=\operatorname{Coker}\left(\pi^{\mathrm{alg}}\left(X_{s}, x\right) \rightarrow \pi^{\mathrm{alg}}(X, x)\right)$ we get a surjective map of group schemes $G \rightarrow \pi^{\text {alg }}(S, s)$. From condition (a) we know the functor

$$
\operatorname{Rep}_{k}\left(\pi^{\mathrm{alg}}(S, s)\right) \rightarrow \operatorname{Rep}_{k}(G)
$$

is essentially surjective, while the surjectivity of $G \rightarrow \pi^{\text {alg }}(S, s)$ tells us that

$$
\operatorname{Rep}_{k}\left(\pi^{\operatorname{alg}}(S, s)\right) \rightarrow \operatorname{Rep}_{k}(G)
$$

is an equivalence of categories. This finishes the proof.

3.3. The general case. In this subsection, we come back to the general settings. All the assumptions are the same as those in $§ 3.1$.

Proposition 3.13. Let $k \subseteq k^{\prime}$ be a field extension. Let $f^{\prime}: X^{\prime} \rightarrow S^{\prime}$ be the base change of the morphism $f: X \rightarrow S$ from $k$ to $k^{\prime}, x^{\prime}, s^{\prime}$ be the base change of $x, s$ respectively. If the sequence of $k^{\prime}$-group scheme homomorphisms

$$
\pi^{a l g}\left(X_{s^{\prime}}^{\prime}, x^{\prime}\right) \rightarrow \pi^{a l g}\left(X^{\prime}, x^{\prime}\right) \rightarrow \pi^{a l g}\left(S^{\prime}, s^{\prime}\right) \rightarrow 1
$$

is exact, then the sequence of $k$-group scheme homomorphisms

$$
\pi^{a l g}\left(X_{s}, x\right) \rightarrow \pi^{a l g}(X, x) \rightarrow \pi^{a l g}(S, s) \rightarrow 1
$$

is also exact.

Proof. Let $\mathfrak{C}\left(X^{\prime}\right)$ be the full subcategory of $\operatorname{Mod}_{c}\left(D_{X^{\prime} / k^{\prime}}\right)$ whose objects are those whose push forward along the projection $X^{\prime} \rightarrow X$ are the inductive limits of their coherent subojects (i.e. subojects belonging to $\operatorname{Mod}_{c}\left(D_{X / k}\right)$ ). This $\mathfrak{C}\left(X^{\prime}\right)$ is a Tannakian subcategory and its Tannaka dual group is precisely $\pi^{\text {alg }}(X, x) \times_{k} k^{\prime}$ [De1, 10.38, 10.41]. But it is clear that this full subcategory is also stable under taking subquotients. Thus the canonical map $\pi^{\text {alg }}\left(X^{\prime}, x^{\prime}\right) \rightarrow \pi^{\text {alg }}(X, x) \times_{k} k^{\prime}$ is surjective. The same argument applies to $X_{s}$ and $S$. Hence we get a commutative diagram with the first row being exact

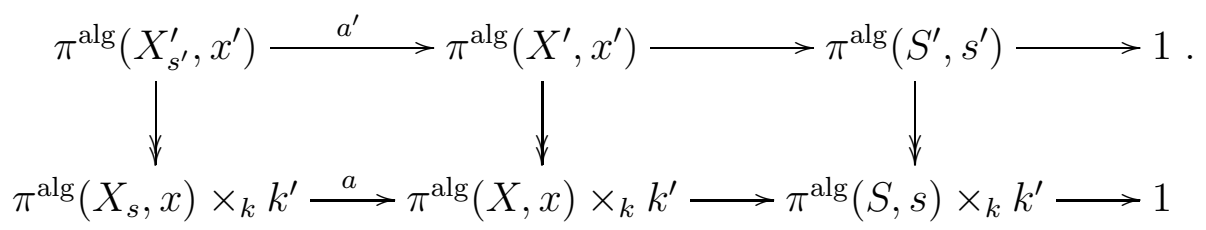

Since the image of $a^{\prime}$ is normal the image of $a$ is normal too. Hence the image of $\pi^{\text {alg }}\left(X_{s}, x\right) \rightarrow \pi^{\text {alg }}(X, x)$ is normal. Using the same argument employed in 3.12 we conclude the proof of this proposition.

Theorem 3.14. Let $f: X \rightarrow S$ be a proper smooth morphism between two smooth connected schemes of finite type over a field $k$ of characteristic 0. Suppose that all the geometric fibres of $f$ are connected. Let $x \in X(k), s \in S(k)$ and $f(x)=s$. Then the homotopy sequence

$$
\pi^{a l g}\left(X_{s}, x\right) \rightarrow \pi^{a l g}(X, x) \rightarrow \pi^{a l g}(S, s) \rightarrow 1
$$

is exact. 
Proof. We have already checked the conditions (a) (b) and surjectivity in $§ 3.1$, so the only thing left is to show condition (c). For any object $\left(E, \nabla_{E}\right) \in \operatorname{Mod}_{c}\left(D_{X / k}\right)$ and any morphism

$$
\delta:\left.\left(F^{\prime}, \nabla_{F^{\prime}}\right) \subseteq\left(E, \nabla_{E}\right)\right|_{X_{s} / k} \in \operatorname{Mod}_{c}\left(D_{X_{s} / k}\right)
$$

there is a finitely generated field over $\mathbb{Q}$ on which all these objects $\left(X, S,\left(E, \nabla_{E}\right), \cdots\right)$ and morphisms $(f, x, s, \delta, \cdots)$ are defined. So we can reduce our problem to the case when $k$ is a finitely generated field over $\mathbb{Q}$. But in light of 3.13 we can assume our field $k$ is actually $\mathbb{C}$.

Let $K$ be the algebraic closure of the function field of $S$. Since $K$ and $\mathbb{C}$ have the same transcendental degree over $\mathbb{Q}$, they are isomorphic as fields. Now $\eta: \operatorname{Spec}(K) \hookrightarrow S$ is a geometric generic point, so by the discussion in $\S 3.2$ the sequence

$$
\pi^{\mathrm{alg}}\left(X_{\eta}, \eta^{\prime}\right) \rightarrow \pi^{\mathrm{alg}}\left(X_{K}, \eta^{\prime}\right) \rightarrow \pi^{\mathrm{alg}}\left(S_{K}, \eta\right) \rightarrow 1
$$

is exact (where $X_{K}$ (resp. $S_{K}$ ) is the base change of $X$ (resp. $S$ ) from $k$ to $K$, and $\eta^{\prime}$ is any chosen $K$-rational point of $X_{K}$ above $\eta$ ). From 3.15 we get a commutative diagram of $K$-group schemes

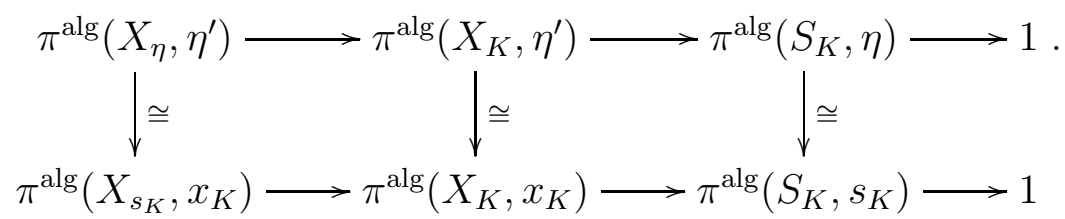

Thus the last row is exact. Then we can conclude our theorem by 3.13 ,

Lemma 3.15. If $f: X \rightarrow S$ is a smooth proper morphism between two smooth quasicompact geometrically connected $\mathbb{C}$-schemes with geometrically connected fibres, $x, x^{\prime}$ and $s, s^{\prime}$ are $\mathbb{C}$-rational points of $X$ and $S$ respectively with the property that $f(x)=s$ and $f\left(x^{\prime}\right)=s^{\prime}$, then there exists a commutative diagram of $\mathbb{C}$-group schemes

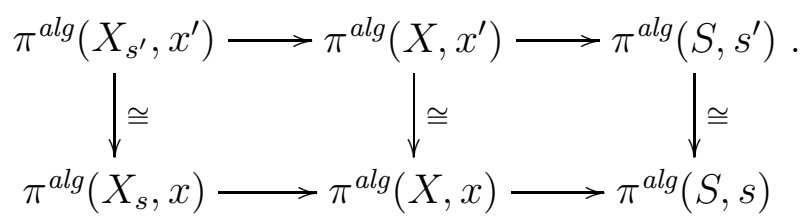

Proof. From the sequences of $\mathbb{C}$-schemes:

$$
X_{s} \rightarrow X \stackrel{f}{\rightarrow} S \quad \text { and } \quad X_{s^{\prime}} \rightarrow X \stackrel{f}{\rightarrow} S
$$

one gets sequences of analytic spaces:

$$
X_{s}^{a n} \rightarrow X^{a n} \stackrel{f^{a n}}{\longrightarrow} S^{a n} \quad \text { and } \quad X_{s^{\prime}}^{a n} \rightarrow X^{a n} \stackrel{f^{a n}}{\longrightarrow} S^{a n},
$$

where $X_{s}^{a n}$ (resp. $X_{s^{\prime}}^{a n}$ ) is still the fibre of $s \in S^{a n}$ (resp. $s^{\prime} \in S^{a n}$ ) under $f^{a n}$, for the functor $-{ }^{a n}$ commutes with fibre product [SGA1, Exposé XII, 1.2]. Now applying the first 
homotopy functor (in topology) to these analytic spaces one gets a commutative diagram:

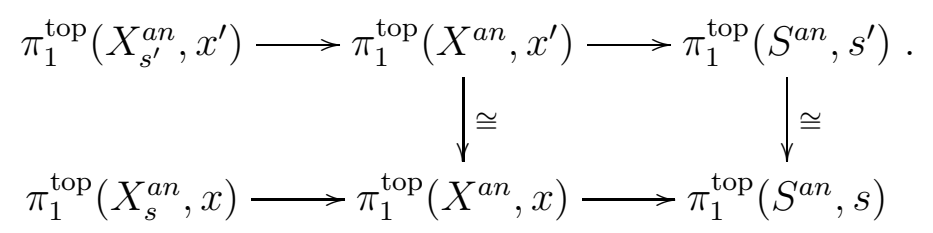

In fact by carefully choosing a path between $x$ and $x^{\prime}$, there exists a group isomorphism

$$
\pi_{1}^{\mathrm{top}}\left(X_{s}^{a n}, x\right) \stackrel{\cong}{\rightarrow} \pi_{1}^{\mathrm{top}}\left(X_{s^{\prime}}^{a n}, x^{\prime}\right)
$$

making the above diagramme commutative.

To show this, one first defines a subset $Z \subseteq S^{a n}$ consisting of points $t \in S^{\text {an }}$ which satisfy that there exists a point $y \in X^{\text {an }}$ lying above $t$, a path $\alpha$ between $x$ and $y$, and an isomorphism

$$
\pi_{1}^{\mathrm{top}}\left(X_{s}^{a n}, x\right) \stackrel{\cong}{\rightarrow} \pi_{1}^{\mathrm{top}}\left(X_{t}^{a n}, y\right)
$$

making the diagram

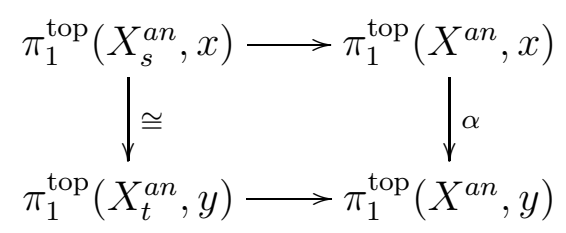

commutative. $Z$ is both open and closed, since for any $t \in S^{a n}$ by Ehresmann's theorem ( $f^{a n}$ is proper smooth by [SGA1, Exposé XII, proposition 3.1 et proposition 3.2]) there exists a neighborhood $U$ of $t \in S^{a n}$ such that $f^{a n-1}(U)$ is isomorphic to $X_{t}^{a n} \times S^{a n}$ as a topological space. Thus for any $t^{\prime} \in U$ one gets a commutative diagram

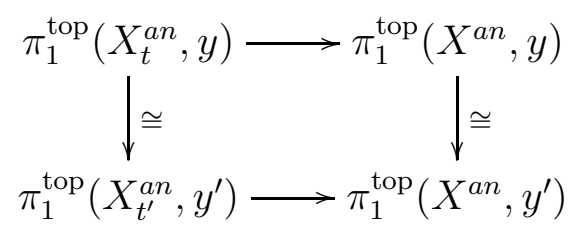

by choosing any points $y, y^{\prime} \in f^{a n-1}(U)$ and any path $y \rightarrow y^{\prime}$ inside $f^{a n-1}(U)$. Hence $t \in Z$ if and only if $t^{\prime} \subseteq Z$. This shows that $Z$ is both open and closed. On the other hand, we know that $S^{a n}$ is connected ([SGA1, Exposé XII, proposition 2.4]). Thus $Z=S^{a n}$ and in particular $s^{\prime} \in Z$.

Now let us denote the category of integrable analytic connections on $X^{\text {an }}$ and $X_{s}^{a n}$, $X_{s^{\prime}}^{a n}$ by $\operatorname{Conn}\left(X^{a n}\right)$ and $\operatorname{Conn}\left(X_{s}^{a n}\right)$, Conn $\left(X_{s^{\prime}}^{a n}\right)$ respectively. By Riemann-Hilbert correspondence one has a 2-commutative diagram of neutral Tannakian categories (the $k$-linear 
tensor functors in the diagram also respect the fibres functors):

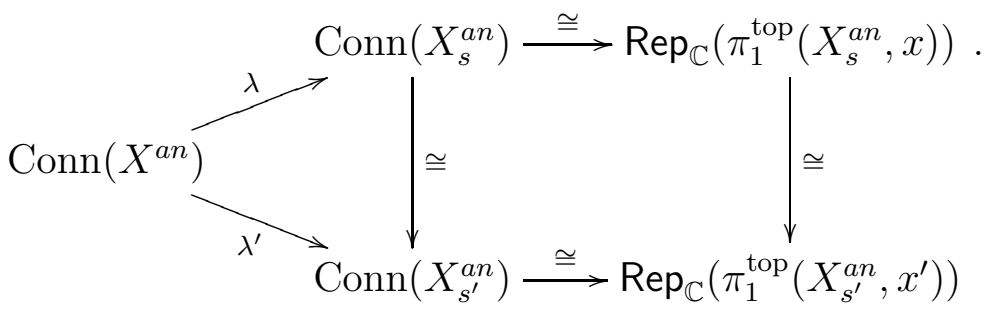

Set $\iota$ to be the canonical functor $\operatorname{Mod}_{c}\left(D_{X / \mathbb{C}}\right) \rightarrow \operatorname{Conn}\left(X^{a n}\right)$ sending an integrable algebraic connection on $X$ to an integrable analytic connection on $X^{a n}$. Then we have a 2-commutative diagram of neutral Tannakian categories:

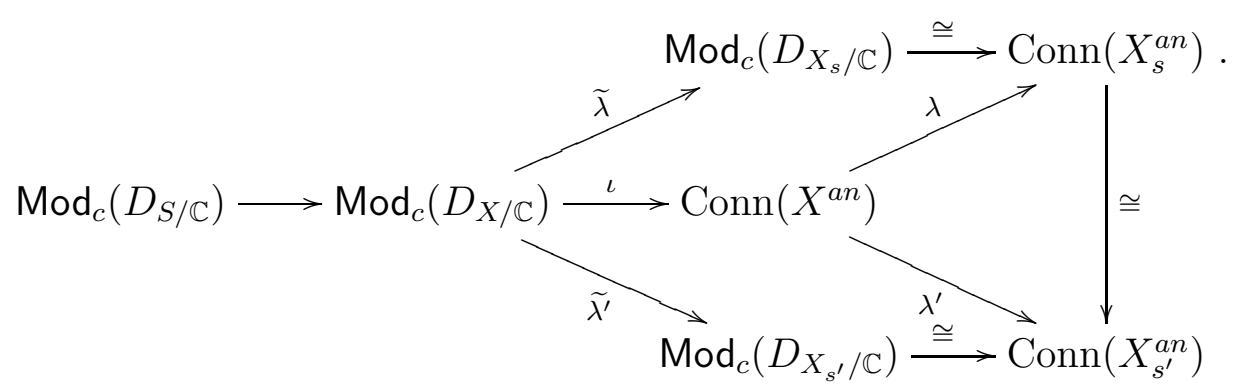

Now applying Tannakian duality we can conclude the proof.

\section{REFERENCES}

[De1] P.Deligne, Le Groupe Fondamental de la Droit Projective Moins Trois Points: in Galois Group over $\mathbb{Q}$, Springer-Verlag, 1989.

[De2] P.Deligne, Catégories Tannakiennes: in The Grothendieck Festschrift, Volume II, Birkäuser, 1990.

[E] H.Esnault., A letter to Phùng Hô Hai (Unpublished), 26-12-2010.

[EP] H.Esnault, P.H.Hai, The Gauss-Manin connection and Tannaka duality, International Mathematics Research Notices Volume 2006, Article ID 93978, Pages 1-35.

[EPS] H.Esnault, P.H.Hai, X.Sun, On Nori's Fundamental Group Scheme, Progress in Mathematics, Vol.265, 366-398, Birkhäuser Verlag Basel/Switzerland, 2007.

[Gies] D.Gieseker, Flat vector bundles and the fundamental group in non-zero characteristic, Ann. Scu. Norm. Sup. Pisa, 4. série, tome 2 (1975), no.1, 1-31. MR0382271 (52 \#3156).

[Katz] N. Katz, Nilpotent connections and the monodromy theorem: applications of a result of Turrittin. IHES, 39, (1970) pp. 175- 232.

[Mats] H.Matsumura, Commutative Ring Theory, Cambrige Studies in Advanced Mathematics, 2000.

[Mum] D.Mumford, Abelian Varieties, Tata Institute of Fundamental Research, Hindustan Book Agency, 2008.

[SGA1] A.Grothendieck, Revêtments Étales et Groupe Fondamental, Springer-Verlag, 1971.

Universität Duisburg-Essen, FB6, Mathematik, 45117 Essen, Germany

E-mail address: lei.zhang@uni-due.de 